\title{
Experiences of Renal Stone Fragmentation with the Use of the Ultrasound-guided Mini-percutaneous Nephrolithotipsy in 650 Patients
}

\author{
Duong Van Trung, Trinh Hoang Hoan, Nguyen Van Hoang, Tran Hong Quan \\ Department of the Urology Surgery, Ha Noi Hospital of Post and Telecommunication, Vietnam
}

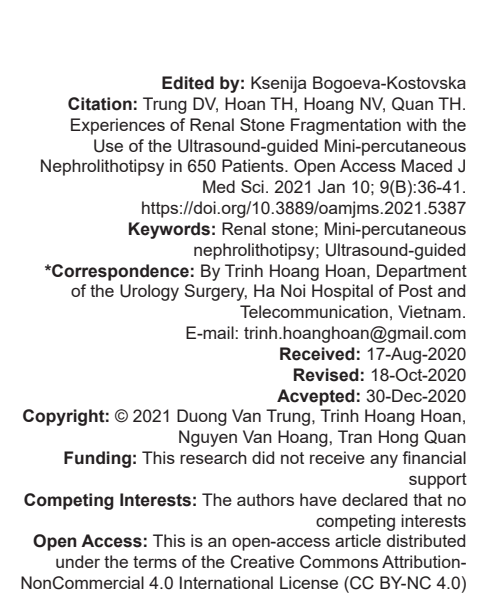

\begin{abstract}
AIM: This study assesses the results of treatment using the mini-percutaneous nephrolithotipsy (PCNL) procedure on renal stone patients in a lateral position under ultrasound guidance, performed at the Ha Noi Hospital of Post and Telecommunications

METHODS: The study was conducted with 650 kidney stone patients who were treated using the ultrasound-guided mini-PCNL procedure in a lateral position, at the Ha Noi Hospital of Post and Telecommunications, over the period from June 2018 to June 2019.

RESULTS: For the 650 patients, the mean age was $47.3 \pm 7.6$ (from 21 to 91 years old); the mean size of stones: $19.4 \pm 1.2 \mathrm{~mm}$ (from $12 \mathrm{~mm}$ to $60 \mathrm{~mm}$ ); the mean operative time: 49.3 minutes (from 37 to $90 \mathrm{~min}$ ); the mean period of hospitalization: 3.9 days (from 3 to 12 days); the mean stone-free rate (SFR): $90.6 \%$; the rate of second surgery: $1.07 \%$; hemorrhage complication: $0.8 \%$; urinary tract infections: $7.7 \%$; septicemia: $0.6 \%$; administered open surgery: $0.46 \%$; and administered other methods: $0.76 \%$.

CONCLUSION: Renal stone fragmentation using the mini-PCNL procedure, performed on patients placed in latera position under ultrasound guidance, is a method that is effective, beneficial, and safe for patients with renal stones and upper ureteral stones.
\end{abstract}

\section{Introduction}

Kidney stones are a common disease, accounted for $5-10 \%$ of the population. Moreover, the relapse rate of the disease is high, possibly up to $50 \%$. Therefore, it badly affects the economy and decreases living standards.

Medical advances have improved the treatment of kidney stones. The minimally invasive methods of treating renal stones, such as extracorporeal shock wave lithotripsy (ESWL), retrograde intrarenal surgery (RIRS), and percutaneous nephrolithotipsy (PCNL), have steadily increased as a replacement for open surgery.

The PCNL method was first performed and introduced by Fernstrom and Johanson in 1976 and has since been studied and developed [1]. PCNL has steadily replaced other methods of treating renal stones; however, with big access tracts required, measuring from 26 to $30 \mathrm{Fr}$, it still causes much injury to the kidneys and affects patients' rehabilitation.

To reduce the negative effects caused by the standard-PCNL, the instrument has been improved in the form of "minimally evasive PCNL" or "mini-PCNL," with access tracts measuring less than 12-20 Fr. This allows more choices for the localization of stone and surgery positions, resulting in less post-operative pain, less bleeding, less potential renal failure, and other kinds of complications; it also is more effective for the patients. In 1997, Helal et al. reported a mini-PCNL operation performed on a baby girl at the age of 2 , in which an access tract measuring up to $16 \mathrm{Fr}$ was used; since then, the mini-PCNL has been performed and developed around the world [2]. In 2012, Abdelhafez et al. conducted a study on the use of the mini-PCNL on 83 patients, which showed a high success rate [3]. In 2013, Zeng et al. examined and summarized 10,000 cases treated with the mini-PCNL; the results showed that the stone-free rate (SFR) in respect of simple calyx of kidney stones was $77.6 \%$, and for complex stones, it was $66.4 \%$ [4]. In 2017, Hennessey et al. reported 30 cases treated using the mini-PCNL, in which the SFR was $96.5 \%$ [5].

In Vietnam, the mini-PCNL was first performed in 2012 and has since been carried out in a large number of health facilities. There have been reports on and assessments of the efficiency and safety scale of this procedure. For instance, in 2018, Thanh et al. reported 
the efficiency of the mini-PCNL treatment for 250 cases at the Vietnamese - Germany Friendship Hospital, with the SFR of $80.7 \%$ [6]. To reduce complications caused by the procedure, we performed the operation in the lateral position, which has the advantages of less effect on the respiration and circulation; this allows it to be carried out on kyphosis sufferers, obese patients, and surgical accidents can be dealt with quickly. Using ultrasound guidance to locate the stone helps the procedure to be carried out simply and avoids the adverse effects of X-ray location on a fluorescent screen.

In our hospital, the standard-PCNL is well performed. In addition, we have been performing the mini-PCNL since 2017. With a view to assessing the efficiency and safety scale of the method, we have conducted this study and reported the results of the ultrasound-guided mini-PCNL performed in a lateral position at the $\mathrm{Ha}$ Noi Hospital of Post and Telecommunications.

\section{Object and Method}

We conducted a prospective, cross-sectional study on 650 patients who were treated using the mini$\mathrm{PCNL}$ in a lateral position under ultrasound guidance; this took place at the $\mathrm{Ha}$ Noi Hospital of Post and Telecommunications from June 2018 to June 2019. The following criteria were used for selecting patients: Size of stone $>2 \mathrm{~cm}$, located at the renal pelvis or calyx of kidney; upper ureteral stones; patients who had undergone a prior failed ESWL or did not agree to be treated with ESWL. Patients with urinary tract infections were cured with antibiotics and excluded serious illness before performing the procedure. All the patients were given clear information and agreed to participate in the study. All the patients were given basic pre-operative tests to assess the location and size of stones, the shape of the calyx of kidney and renal pelvis, as well as other overall tests such as hematology and biochemical blood. One month after the surgery, all the patients underwent a radiography or ultrasound scan and were re-examined.

\section{Operation techniques}

First, the patient was general anesthesia, placed in the obstetric position where a performed ureteroscopy and intubated $7 \mathrm{Fr}$ ureter catheters into the renal pelvis. The patient was then turned $90^{\circ}$ to the lateral position, and the stones' location was identified. A needle measuring $20 \mathrm{~cm}$ was punctured through the calyx of kidney or renal pelvis to the stone: The target signal was a urine flow through the needle or injected sterile saline through ureter catheter and the water flowed through the needle afterward, the feeling of needle touched into the stones. Then, PCNL guide wire was placed through the exploration needle. An incision of $6-7 \mathrm{~mm}$ in length was made, and then the tunnel was dilated by the access tracts from 8 to $18 \mathrm{Fr}$. An Amplatz sheath was introduced into the calyx of kidney - renal pelvis. We examined the calyx of kidney and renal pelvis through the Amplatz sheath to determine the location of the stone. The stone fragmentation was performed by laser at an $80 \mathrm{~W}$ power setting. The stone fragment removal was carried out through the Amplatz. Having checked the calyx of kidney and renal pelvis, a double-J stent was placed between the renal pelvis - ureter. A plastic tract of $16 \mathrm{Fr}$ was then inserted for kidney drainage.

\section{Criteria for assessing stone-free status}

According to the 2015 Guideline of the European Association of Urology (EAU), the stone clearance status was defined as when no residual stones were detected on plain X-ray photographs of the urinary system after the surgery, or there were residual stone fragments of less than $4 \mathrm{~mm}$ in size. "Residue of stone" status refers to cases where one or more stone fragments of more than $4 \mathrm{~mm}$ in size were detected, and the patient continued to be treated in association with other methods [7]. In this study, we assessed the stone-free status based on the post-operative tests, including an ultrasound scan and radiography.

\section{Data handling and collection}

The data were collected and processed by SPSS 20.0, using a statistical algorithm. The t-test and Fisher were used to verify the difference. A comparative value was considered statistically different when $\mathrm{p}<0.05$.

\section{Study Result}

The results achieved by the study on 650 patients treated with the ultrasound-guided mini-PCNL in the lateral position are as follows:

The mean age of the study group was $47.3 \pm$ 7.6 (21-91), of whom the eldest patient was 91 years old. The mean size of stone was $19.4 \pm 1.2(12-60)$, of which the biggest was $60 \mathrm{~mm}$. Cases of a single stone of the renal pelvis were the most common, rated $30 \%$; the rate of calyx of kidney stone was $28.2 \%$; the rate of staghorn stone was $16 \%$; and the rate of multi-position stone accounted for $10 \%$. Among those treated with this procedure, there were 3 of our research patients $(0.5 \%)$ had only 1 kidney; 15 patients $(2.3 \%)$ had undergone renal stone removal surgery before. 
Hydronephrosis degree 1 accounted for $30.7 \%$. We saw a large number of patients with hydronephrosis degree $2(45.5 \%)$, while patients with non-hydronephrosis accounted for $10.5 \%$.

The middle calyx of kidney was the most common renal puncture site, accounting for $57 \%$; the lower calyx of kidney accounted for $39.8 \%$; and the upper calyx of kidney $3.2 \%$ only.

The signs used to definitely determine that the needle had entered the calyx of kidney were when it touched the stones (accounting for 76.1\%); liquid leaked out when sucked by a syringe (5.5\%), and the failure rate of being unable to puncture the calyx of kidney and renal pelvis was 6 patients $(0.9 \%)$.

In the study, the number of failures was 8 patients, of which 6 cases related to the failure to puncture, and 2 patients were successfully punctured but bled when tunnels were created. There were 3 patients who had to be administered open surgeries $(0.46 \%), 3$ patients who had to be administered endoscopy operations $(0.46 \%)$, and 2 patients who had to be given double-J stents and administered ESWL $(0.3 \%)$.

In the 642 patients successfully treated with the mini-PCNL, the mean operative time was 49.3 (3790) min: The shortest case was $37 \mathrm{~min}$ and the longest was $90 \mathrm{~min}$.

The upper ureteral stones and single renal pelvic stone accounted for a high SFR, 100\% and $98.9 \%$, respectively. A simple calyx of kidney stone had a relatively high SFR of $95.6 \%$. For multiple stone renal pelvis and calyx of kidney, the SFR was very low (39\%), of which 7 patients $(1.07 \%)$ were administered a second mini-PCNL. The difference was statistically significant at $p<0.05$.

There were 3 patients bleeding that needed vascular interventional therapy after the procedure and well discharged. The most common complications were urinary infection, accounting for $7.7 \%$, and septicemia found in 4 patients $(0.6 \%)$, who were then given a test for blood culture and treated with antibiotics according to the treatment regimen.

The mean period of hospitalization was 3.9 (3-12) days. The longest period was 12 days, seen in the patient with septicemia, who was then treated with antibiotics.

\section{Discussion}

Urinary stones are a common disease throughout the world; according to the EAU in 2015, the risk of an individual developing a kidney stone during their life is about $5-10 \%$. Among patients with urinary stones, kidney stones account for about $70-75 \%$ at the age of 30-60 [7]. In our study, the mean age of patients was $47.3 \pm 7.6$ (21-91) (Table 1). A couple of other studies have given the same results; for instance, Zimmermanns et al. reported on 652 patients with the mean age of $54.1 \pm 15.7$, and Zare studied 250 patients with the mean age of $42.0 \pm 13.4$ [8], [9]

Table 1: Patient information before PCNL $(n=650)$

\begin{tabular}{lll}
\hline Indicators & Number of patients & $\%$ \\
\hline Mean age & $47.3 \pm 7.6(21-91)$ & \\
Mean size of stone (mm) & $19.4 \pm 1.2(12-60)$ & \\
Location of stone & 103 & 15.8 \\
$\quad$ Upper ureteral stones & 195 & 30 \\
Renal pelvis stone & 183 & 28.2 \\
Calyx of kidney stone & 104 & 16 \\
Staghorn stone & 65 & 10 \\
$\quad$ Multi-position stone & 68 & 10.5 \\
Hydronephrosis & 200 & 30.7 \\
$\quad$ Non-hydronephrosis & 296 & 45.5 \\
Degree 1 & 86 & 13.3 \\
Degree 2 & & \\
Degree 3 & & 10 \\
\hline
\end{tabular}

The mean size of stone in our study was 19.4 $\pm 1.2(12-60) \mathrm{mm}$ (Table 1). According to the (EAU, 2015), the mini-PCNL is recommended as a choice of treatment for big stones (>20 mm) and smaller stones (10-20 mm), where treatment failed in ESWL or ESWL was contraindicated [7].

The common location of stones in our study was renal pelvic stones, accounting for $30 \%$; simple calyx of kidney stone $28.2 \%$; staghorn stones $16 \%$; and multiple stones renal pelvis and calyx of kidney accounted for $10 \%$. We especially indicated the miniPCNL procedure for patients with large upper ureteral stones (>15 mm) or who had undergone a failed ESWL; this group accounted for $15.8 \%$ (Table 1). According to Abdelhafez et al. study in 2012, on 83 patients, simple stones accounted for $38.6 \%$; multiple, complex stones accounted for $61.4 \%$ [3]. Zare recorded $62 \%$ of complex stones, $12 \%$ of pelvic renal stones, and $4 \%$ of upper ureteral stones [9]. Hydronephrosis degrees 1, 2, and 3 for the patients in our study group were $30.7 \%, 45.5 \%$, and $13.3 \%$, respectively (Table 1 ). In Zare's study, the respective degrees of hydronephrosis was $23.2 \%$, $16.8 \%$, and $7.2 \%$ [9].

In the mini-PCNL procedure, we chose the lateral position under the ultrasound guidance, in accordance with clinical facts and previous studies.

The comparative study by Basiri et al. on 100 patients, using ultrasound guidance and fluoroscopic guidance, has given the same result but affirmed that the ultrasound guidance helped to avoid radiation [10]. In 2015, Wang's study reported on 3019 patients, 1574 of whom were operated on using ultrasound guidance and 1445 under fluoroscopic guidance; it concluded that apart from the X-ray exposure, the patients under ultrasound guidance had a reduced blood loss rate, a reduced rate of complications, a shorter time of tunnel creation, and a higher SFR [11]. Knoll et al. indicated that because the ultrasound guidance helped to easily observe internal organs, therefore, damage to the large intestine, liver, or spleen could be avoided [12]. Pan et 
al. studied 100 patients, divided into two groups: The prone and lateral positions. They concluded that both positions affected the circulation and arterial blood gas; however, the lateral position saw smaller changes of blood gas and hemodynamics [13]. The lateral position was familiar, allowing the surgeon to quickly deal with intra-operative complications.

The localization of the puncture site is of importance when performing the mini-PCNL procedure, as it ensures better control and stone clearance, as well as a reduced risk of complications. The localization of the puncture site depends on the location and size of stones and also the complexion of the urinary system. The most appropriate puncture line is that which least damages the veins and internal organs, which is the perpendicular line to the backside outside the kidney, into Brodel's bloodless line. This puncture line will approach mainly the middle and lower calyxes. In our study, the middle calyx of kidney puncture accounted for $57 \%$, the lower calyx of kidney accounted for $39.8 \%$, and the upper calyx of kidney accounted for $3.2 \%$ (Table 2). The advantage of the middle calyx of kidney puncture is that it helps to effectively monitor the calyx of kidney and renal pelvis.

Table 2: Process of needle exploration $(n=650)$

\begin{tabular}{llll}
\hline Indicators & Variables & Patients & Rate (\%) \\
\hline Positions & Upper calyx of kidney & 21 & 3.2 \\
& Middle calyx of kidney & 370 & 57 \\
Signals the & Lower calyx of kidney & 259 & 39.8 \\
exploration needle & Needle touching the stone & 495 & 76.1 \\
was in the calyx of & Suck the wale saline through ureter catheters & 114 & 17.5 \\
kidney & Exploration failure & 35 & 5.5 \\
Result & Success & 6 & 0.9 \\
& Failure & 642 & 98.8 \\
Time & Mean operative time & 8 & 1.2 \\
\hline
\end{tabular}

In Zare's study, the middle calyx of kidney puncture accounted for $57.2 \%$, the lower calyx of kidney puncture $33.2 \%$, and the upper calyx of kidney $9.6 \%$ [9]. In 2012, Abdelhafez et al. reported on 83 patients treated with the mini-PCNL, in which the upper calyx of kidney puncture accounted for $4.8 \%$, the middle calyx of kidney puncture $10.8 \%$, and the rest was the lower calyx of kidney [3]. Hennessey et al. studied 32 cases treated using the mini-PCNL, of which 24 cases $(75 \%)$ were punctured at the lower calyx of kidney, $12.5 \%$ at the middle calyx of kidney, and $12.5 \%$ the upper calyx of kidney [5]. For simple stones, using the calyx of kidney, renal pelvis puncture to directly approach the stones is the best way to the stone fragmentation and to limit damage and complications. We carried out the puncture to directly approach the location of the stone at the calyx of kidney, using the sign of the needle touching the stones in $76.1 \%$; for patients whose calyces of kidney and renal pelvis were not dilated, we pumped physiological saline through the urinary tract, and determined it was successful when water flowing through the needle was observed, or water flowing out when directly sucked by a syringe through the needle; these groups accounted for $17.5 \%$ and $5.5 \%$, respectively (Table 2 ). Hydronephrosis greatly affects the process of puncturing and creating the tunnel. All the cases with hydronephrosis degrees 2 and 3 were successfully punctured. In the study group, there were 9 patients $(0.9 \%)$ who were unsuccessfully punctured, and we failed to approach the stone; here, we had recourse to other methods, such as open surgery (1 case), endoscopy surgery (3 cases), and ESWL (2 cases) (Table 1). These cases of failed puncture were seen in those with hydronephrosis degree 1 and non-hydronephrosis. In addition, there were 2 patients whose stones were approached, but bleeding occurred while performing the procedure, and therefore open surgery had to be administered to remove the stone and stop the bleeding. For these cases, where there were large, complex stones occupying different locations, the mini-PCNL had a low SFR; therefore, we chose open surgery to ensure safety and the stone clearance. Do et al. studied 250 patients, in which 3.6\% of procedures had to cease due to operative bleeding, and a second mini-PCNL was then carried out; no case was administered an open surgery [6].

Among 642 patients who were successfully punctured, with a tunnel created and the kidney approached, the mean operative time was 49.3 (37-90) min, and the longest was $90 \mathrm{~min}$, recorded in the case with staghorn stones (Table 2). According to Hennessey et al. (2017), for 32 patients, the mean operative time was 50 (40-82) min, with the mean size of stones equivalent to our study 17 [5]. The operative time was linked with the location and size of the stones, according to Abdelhafez et al. (2012), who studied the treatment by mini-PCNL with the mean size of stones of $36.7 \pm 23.37 \mathrm{~mm}$ : In this case, the mean operative time was $99.2 \pm 48.3$ minutes [3]

\section{SFR}

In our study group (642 patients), the mean SFR was $90.6 \%$, of which all patients with upper ureteral stones showed a full SFR (100\%); the patients with a simple renal pelvic stone showed a SFR of $98.9 \%$; the patients with calyx of kidney stones showed a SFR of $95.6 \%$; those with staghorn stones recorded a SFR of $89.3 \%$; and patients with multiple, complex stones occupying the calyx of kidney; and renal pelvis showed the lowest SFR of $39 \%$. Only $1.07 \%$ of cases were administered a second mini-PCNL (Table 3). There was a difference between simple stones and complex stones (multiple stone renal pelvis and calyx of kidney, staghorn stones); it was considered statistically significant at $p<0.05$. Abdelhafez et al. (2012) found that

Table 3: Rate of stone clearance $(n=642)$

\begin{tabular}{llll}
\hline Stone location & Total number of patients & Stone-free & Rate (\%) \\
\hline Upper ureteral stones & 103 & 103 & 100 \\
Renal pelvis stone & 192 & 190 & 98.9 \\
Calyx of kidney stone & 180 & 172 & 95.6 \\
Staghorn stone & 103 & 92 & 89.3 \\
$\begin{array}{l}\text { Multiple stone renal pelvis and } \\
\text { calyx of kidney }\end{array}$ & 64 & 25 & 39.0 \\
Total & 642 & 582 & 90.6 \\
\hline
\end{tabular}


for 83 cases treated with the mini-PCNL, the SFR was $78.3 \%$ at the first operation, 14 cases $(16.9 \%)$ needed other methods, and 4 cases $(4.8 \%)$ were administered a second mini-PCNL. There was a significant difference in the SFRs between simple stones and staghorn stones: $96.9 \%$ as compared with $66.7 \%$ ( $p<0.05$ ), respectively [3]. For upper ureteral stones $(>15 \mathrm{~mm})$, the SFR was $100 \%$. We chose the mini-PCNL procedure for this type of stone, instead of the RIRS, to limit the rate of residual stones. The advantages of the miniPCNL over the RIRS in the treatment of upper ureteral stones ( $\geq 15 \mathrm{~mm}$ ) were studied by Gu et al. (2013), who found the SFRs of $93.3 \%$ and $41.4 \%$, respectively [14].

According to Abdelhafez et al. report (2013), the mini-PCNL seems more effective for the treatment of smaller stones ( $<20 \mathrm{~mm}$ ) as compared with bigger stones $(>20 \mathrm{~mm}$ ), with the SFRs of $90.8 \%$ and $76.3 \%$, respectively [15]. Zeng's study showed the same result, with the SFR for simple stones and complex stones being $77.6 \%$ and $66.4 \%$, respectively $(p<0.05)$. The difference gradually decreased for second mini-PCNL treatments or other interventions, showing the SFRs of $86.7 \%$ and $86.1 \%$ ( $p>0.05$ ) [4]. According to Ozgor et al., who studied 360 cases with caliceal stones, the SFR after mini-PCNL treatment reached $84.7 \%$ [16].

\section{Complications}

The post-operative complication rate of our study group was $8.8 \%$, mostly consisting of fever caused by infections, of which urinary infection was contracted by $7.7 \%$. These patients underwent urine tests and were given antibiotics according to the treatment regimen. The rate of blood infection was $0.6 \%$, and these patients were also given antibiotics according to the treatment regimen, producing a good result. The urethra catheters, kidney drainage bleeding occurred in 3 patients $(0.5 \%)$ in the post-operative period; their veins were X-rayed, and vascular interventional, resulting in good progress (Table 4). Hennessey et al. studied 32 cases given the mini-PCNL treatment and found that 3 cases $(9.3 \%)$ developed complications: 1 patient contracted urinary infection $(3.1 \%), 1$ patient had an injured vein and was administered internal medicine, 1 patient developed septic shock and received intensive care [5]. When studying 250 patients, Thanh et al. found that post-operative complications occurred in $5.2 \%$ of cases, including 1 case of hemorrhage that was managed by vascular interventional, 8 cases of fever caused by infection that was given antibiotic, and 3 cases of septic shock that was managed by intensive care [6]. In our study, no case of complication related to

Table 4: Post-operative care $(n=642)$

\begin{tabular}{|c|c|c|c|}
\hline Indicators & Variables & Patients & Rate $(\%)$ \\
\hline \multirow[t]{3}{*}{$\begin{array}{l}\text { Post-operative } \\
\text { complications }\end{array}$} & $\begin{array}{l}\text { Bleeding that needed vascular interventional } \\
\text { therapy after the procedure }\end{array}$ & 3 & 0.5 \\
\hline & Fever, urinary infection & 50 & 7.7 \\
\hline & Fever, septicemia & 4 & 0.6 \\
\hline Time & Mean period of hospitalization & $3.9(3-12$ & days \\
\hline
\end{tabular}

the digestive system or pleural lesions was observed. This result was partly due to the experiences of the surgeon and partly because of the lateral position and the ultrasound guidance, which helped to reduce the related complications. According to Zare, who studied 250 patients treated with the mini-PCNL, the rate of post-operative infection was $10 \%$, of which $0.4 \%$ were pleural lesions that needed surgical interventions [8].

The mean length of hospitalization of the study group was 3.9 (3-12) days; the longest stay was observed for the patient with septicemia who was given antibiotics according to the treatment regimen (Table 4). Thanh et al. studied 250 patients and found that the mean length of hospitalization was $4.57 \pm 2.64$ days [6]. The study by Mishra et al. indicated that the lengths of hospital stay of the mini-PCNL were $3.2 \pm 0.8$ days [17].

\section{Conclusion}

Through this study, we have demonstrated that the Ultrasound-guided Mini-PCNL in the Lateral Position is an effective and safe method, especially for upper ureteral stones (>15 mm) and simple stones. The SFR of the mini-PCNL procedures increases when associated with other methods of intervention. As this procedure produces the advantages of high SFR and radiation avoidance, and with fewer complications, it is highly recommended that the mini-PCNL be continued, improved, and widely applied at health facilities, to bring benefits for patients.

\section{References}

1. Fernstrom I, Johansson B. Percutaneous pyelolithotomy. A new extraction technique. Scand J Urol Nephrol 1976;10(3):257-9. PMid:1006190

2. Helal M, Black T, Lockhart J, Figueroa TE. The Hickman peel-away sheath: alternative for pediatric percutaneous nephrolithotomy. J Endourol 1997;11(3):171-2. https://doi. org/10.1089/end.1997.11.171

PMid:9181444

3. Abdelhafez MF, Bedke J, Amend B, EIGanainy E, Aboulella H, Elakkad M, et al. Minimally invasive percutaneous nephrolitholapaxy (PCNL) as an effective and safe procedure for large renal stones. BJU Int 2012;110(11 Pt C):E1022-6. https://doi.org/10.1111/j.1464-410x.2012.11191.x

PMid:22540846

4. Zeng G, Zhao Z, Wan S, Mai Z, Wu W, Zhong W, et al. Minimally invasive percutaneous nephrolithotomy for simple and complex renal caliceal stones: A comparative analysis of more than 10,000 cases. J Endourol 2013;27(10):1203-8. https://doi. org/10.1089/end.2013.0061

PMid:23924320

5. Hennessey DB, Kinnear NK, Troy A, Angus D, Bolton DM, 
Webb DR. Mini PCNL for renal calculi: does size matter? BJU Int 2017;119 Suppl 5:39-46. https://doi.org/10.1111/bju.13839 PMid:28544299

6. Thanh DT, Dang LH, Hoang NH. Ultrasound guided access during percutaneous Nephrolithotomy on patients in lateral position assessment of Outcome in 250 cases traited at the viet-germany friendship Hospital, Vietnam. Int Braz J Urol 2018;7(1):22-30 PMid:27583353

7. Turk C, Petrik A, Sarica K, Seitz C, Skolarikos A, Straub M, et al. EAU guidelines on interventional treatment for urolithiasis. Eur Uro 2016;69(3):475-82. https://doi.org/10.1016/j.eururo.2015.11.009 PMid:26344917

8. Zimmermanns, V., S. Kurzidim, P. Liske, S.Lahme. Minimal invasive PCNL (MPCNL) proven efficiency and safety after more than 650 consecutive patients. Eur Urol Suppl 2012;11(1):e1032. https://doi.org/10.1016/s1569-9056(12)61028-8

9. Zare S. Feasibility of pure ultrasonography guided percutaneous nephrolithotomy in flank position. Nephro Urol Mon 2017;9:e47051. https://doi.org/10.5812/numonthly.47051

10. Basiri A, Ziaee AM, Kianian HR, Mehrabi S, Karami H, Moghaddam SM. Ultrasonographic versus fluoroscopic access for percutaneous nephrolithotomy: A randomized clinical trial. J Endourol 2008;22:281-4. https://doi.org/10.1089/end.2007.0141

11. Wang $\mathrm{K}$, Zhang $\mathrm{P}, \mathrm{Xu} \mathrm{X}$, Fan M. Ultrasonographic versus fluoroscopic access for percutaneous nephrolithotomy: A meta-analysis. Urol Int 2015;95:15-25. https://doi. org/10.1159/000369216

12. Knoll T, Michel MS, Alken P. Surgical atlas percutaneous nephrolithotomy: The mannheim technique BJU Int 2007;99:213-31.https://doi.org/10.1111/j.1464-410x.2007.06613.x

13. Pan T, Liu B, Wei S, Li G, Wen H, Shen G, et al. Flanksuspended versus prone percutaneous nephrolithotomy: changes of haemodynamics, arterial blood gases and subjective feelings. Urologia 2015;82(2):102-5. https://doi. org/10.5301/uro.5000108

PMid:25791394

14. Gu XJ, Lu JL, Xu Y. Treatment of large impacted proximal ureteral stones: Randomized comparison of minimally invasive percutaneous antegrade ureterolithotripsy versus retrograde ureterolithotripsy. World J Urol 2013;31(6):1605-10. https://doi. org/10.1007/s00345-013-1026-2

PMid:23334470

15. Abdelhafez MF, Amend B, Bedke J, Kruck S, Nagele U, Stenzl A, et al. Minimally invasive percutaneous nephrolithotomy: A comparative study of the management of small and large renal stones. Urology 2013;81(2):241-5. https://doi.org/10.1016/j. urology.2012.09.030

16. Ozgor F, Kucuktopcu O, Simsek A, Sarılar O, Binbay M, Gürbüz G. Percutaneous nephrolithotomy for isolated calyceal stones: How important is the stone location? Turk J Urol 2015;41(4):171-6. https://doi.org/10.5152/tud.2015.06787 PMid:26623144

17. Mishra S, Sharma R, Garg C, Kurien A, Sabnis R, Desai M Prospective comparative study of miniperc and standard PNL for treatment of 1 to $2 \mathrm{~cm}$ size renal stone. BJU Int 2011;108(6):896-9; discussion 899-900. https://doi. org/10.1111/j.1464-410x.2010.010284.x

PMid:21477212 